\title{
Making sense of spike D614G in SARS-CoV-2 transmission
}

\author{
Aria C. Shi ${ }^{1} \&$ Xuping $\mathrm{Xie}^{2^{*}}$ \\ ${ }^{1}$ School of Medicine, Johns Hopkins University, Baltimore, MD 21205, USA; \\ ${ }^{2}$ Department of Biochemistry and Molecular Biology, University of Texas Medical Branch, Galveston, TX 77550, USA
}

Received December 14, 2020; accepted January 25, 2021; published online February 4, 2021

\begin{abstract}
Severe acute respiratory syndrome coronavirus 2 (SARS-CoV-2), the etiologic agent of the current coronavirus disease 2019 (COVID-19) pandemic, has evolved to adapt to human host and transmission over the past 12 months. One prominent adaptive mutation is the asparagine-to-glycine substitution at amino acid position 614 in the viral spike protein (D614G), which has become dominant in the currently circulating virus strains. Since spike protein determines host ranges, tissue tropism, and pathogenesis through binding to the cellular receptor of angiotensin converting enzyme 2 (ACE2), the D614G mutation is hypothesized to enhance viral fitness in human host, leading to increased transmission during the global pandemic. Here we summarize the recent progress on the role of the D614G mutation in viral replication, pathogenesis, transmission, and vaccine and therapeutic antibody development. These findings underscore the importance in closely monitoring viral evolution and defining their functions to ensure countermeasure efficacy against newly emerging variants.
\end{abstract}

\section{SARS-CoV-2, D614G, COVID-19}

Citation: Shi, A.C., and Xie, X. (2021). Making sense of spike D614G in SARS-CoV-2 transmission. Sci China Life Sci 64, 1062-1067. https://doi.org/10.1007/ s11427-020-1893-9

\section{Introduction}

The coronavirus disease 2019 (COVID-19), caused by severe acute respiratory syndrome coronavirus 2 (SARS-CoV2), has swept across the world since 2019 (Huang et al., 2020; Zhu et al., 2020). As of December 2020, there have been over 71.2 million cases and nearly 1.6 million global deaths (coronavirus.jhu.edu/map). Although most infections are asymptomatic or develop mild diseases, some patients can develop life-threatening pneumonia, particularly in the elderly and those with chronic comorbidities. The exact mechanism that drives the COVID-19 pathogenesis remains to be defined, though dysregulation and hyperinflammatory responses are known to be responsible for the development of severe respiratory symptoms. SARS-CoV-2 is closely related to a horseshoe bat coronavirus isolate RaTG13, with 96.2\% overall genome sequence identity (Zhou et al.,

*Corresponding author (email: xuxie@utmb.edu) 2020b), suggesting bats as the host origin. However, intermediate hosts responsible for the switch of SARS-CoV-2 from bats to humans have yet to be identified. Upon host switch, viruses must mutate to improve their fitness in the new host. This is particularly true for RNA viruses, which usually lack proofreading capability during replication. Compared with other RNA viruses, coronaviruses have a proofreading mechanism that is required to maintain the fidelity of their large RNA genomes (Smith et al., 2013). However, the proofreading function does not eliminate mutations during coronavirus replication. Thus, it is not surprising that adaptive mutations have occurred in SARS-CoV2 during its continuous replication and transmission in humans over the past year.

\section{Emergence of spike D614G substitution}

For SARS-CoV-2, all original viral isolates reported in 
January 2020 have an Asparagine at amino acid position 614 in the spike protein (D614). This residue has been gradually replaced with the glycine (G614) over time (Figure 1A). Among globally sequenced strains of SARS-CoV-2, the D614G substitution was rare before March 2020, with less than $10 \%$ in frequency, but the D614G frequency has since increased to over $75 \%$ after June (Korber et al., 2020). Transitions from D614 to G614 occurred asynchronously in different regions throughout the world, including even local epidemics where the wildtype (WT) D614 virus was well established prior to the emergence of the G614 variant (Korber et al., 2020). This indicates that the D614G substitution is not due to an incidental founder effect or genetic drift. Clinically, there has been no evidence of change in disease severity associated with the D614G substitution. However, higher levels of viral RNA were detected in nasopharyngeal swabs from the G614 virus-infected patients than those from the D614 virus-infected individuals (Lorenzo-Redondo et al., 2020), suggesting that the D614G substitution may increase viral fitness in humans without causing more severe disease.

\section{Enhanced viral replication in cell culture}

Three groups have independently characterized the biological function of D614G mutation in viral replication, pathogenesis, and transmission using isogenic D614 and G614 SARS-CoV-2 (Hou et al., 2020a; Plante et al., 2020; Zhou et al., 2020a). The isogenic D614 and G614 virus were prepared using reverse genetic systems (Hou et al., 2020b; Xie et al., 2020). Plante et al. first reported that the D614G mutation did not affect the cleavage of full-length spike protein to S1 and S2 subunits; however, the G614 variant enhanced viral replication in human lung epithelial Calu-3 cells and primary human airway tissues (Figure 1B) (Plante et al., 2020). The increased viral replication was much more dramatically observed in the primary airway cultures than that in the Calu-3 cells, particularly when the replication kinetics of the two viruses were compared head-to-head in a competition assay (Hou et al., 2020a; Plante et al., 2020; Zhou et al., 2020a). Although the D614G mutation increased the production of infectious virus, it did not increase the viral RNA levels of the progeny viruses; this led to a lower genomic RNA to plaque-forming unit (PFU) ratio for the G614 virus. The lower genomic RNA to PFU ratio indicates an increased infectivity of the G614 virus (Plante et al., 2020). Besides increased virion infectivity, the D614G mutation improves the thermal stability of SARS-CoV-2. When incubated at $33^{\circ} \mathrm{C}$ (mimicking the upper respiratory temperature), $37^{\circ} \mathrm{C}$, or $42^{\circ} \mathrm{C}$, the G614 virus retains higher infectivity over time than the D614 virus (Plante et al., 2020). The increased infectivity and thermal stability may account for the enhanced viral replication in cell culture and in animal models (see below).

\section{Enhanced viral replication and transmission in animal models}

Hamsters are highly susceptible to SARS-CoV-2 infection and are used as a model resembling fulminant COVID-19 disease in humans (Chan et al., 2020; Imai et al., 2020). In the hamster model, infection with D164 or G614 virus caused equivalent amounts of weight loss; the infected animals exhibited similar levels of disease, supporting the mutation does not affect disease severity. However, the D614G mutation conferred a higher level of viral replication in the upper airway (i.e., nasal wash and trachea) but not in the lower airway (i.e., lung) (Figure 1C) (Plante et al., 2020). Similarly, the D614G mutation also enhanced viral replication in human ACE2 knock-in mice (Zhou et al., 2020a). Two groups have conducted direct viral transmission in hamsters using pairs of infected donor and naive recipient animals housed in neighboring cages (Hou et al., 2020b) or co-housed in the same cage (Zhou et al., 2020a). In hamsters with donors inoculated either with D614 or G614 virus, the G614-infected pairs had earlier transmission than the WT D614-infected pairs (Hou et al., 2020b). In donor hamsters inoculated with an equal infectious ratio of D614 and G614 viruses, the viral RNA from nasal washings of recipient hamsters was composed of over 90\% G614 virus (Zhou et al., 2020a). Similar trends were also observed in competition transmission experiments using ferrets, which models mild human infection (Zhou et al., 2020a). These studies have consistently demonstrated that the D614G mutation increases viral replication in the upper airway, leading to enhanced transmissibility in animal models, but does not affect disease severity. Overall, the animal results recapitulate the clinical findings that the D614G mutation enhances viral RNA level in nasopharyngeal swabs without increasing disease severity. The lack of enhanced replication of G614 virus in the lower airway may explain why differences in clinical severity were negligible when patients were infected with the mutated variant.

\section{Effect on spike structure and ACE2 receptor binding}

Spike protein allows SARS-CoV-2 to enter host cells through interactions with the ACE2 receptor (Shang et al., 2020). The spike protein is glycosylated and forms a homotrimer (Figure 1D). Each spike molecule consists of an N-terminal S1 subunit and a C-terminal S2 subunit, separated by a furin (S1/S2 cleavage) and other protease (S2') 

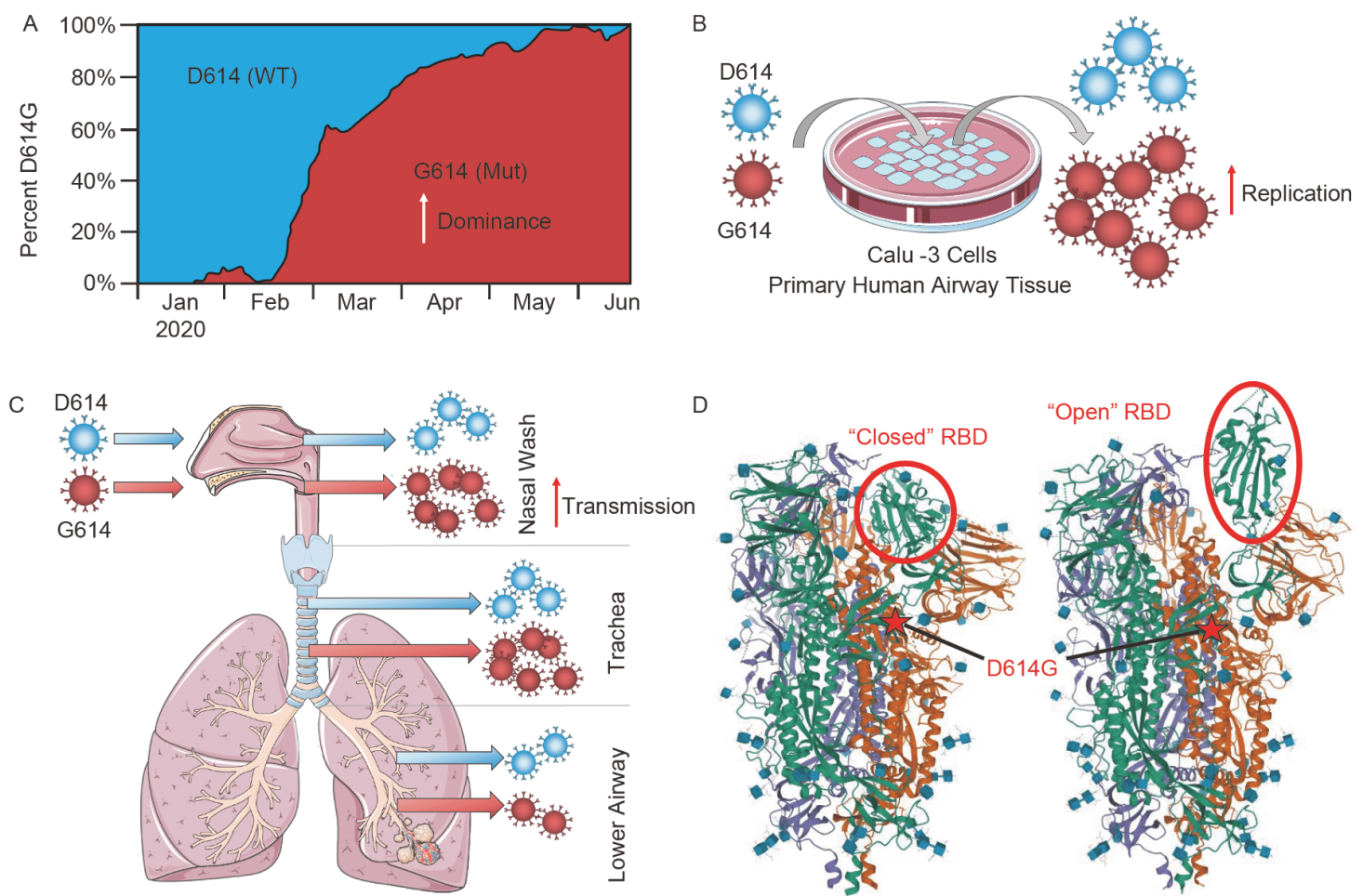

Figure 1 A glance of the epidemiology, experimental evidence and molecular basis of SARS-CoV-2 D614G. A, Emergence of spike D614G substitution. The original SARS-CoV-2 sequences reported in January 2020 had a D614 amino acid in the spike protein, but has been gradually replaced by a D614G substitution, with the G614 variant now dominant throughout the world. Graph is represented with modifications using data from Korber et al. (2020). B, G614 has enhanced viral replication in cell culture. The G614 variant has increased viral replication in human lung epithelial Calu-3 cells and primary human airway tissues. The G614 variant has a lower genomic RNA to PFU ratio, indicating increased infectivity with the D614G substitution. The D614G substitution has also increased the thermal stability of the SARS-CoV-2. C, G614 has enhanced viral replication in the upper airways and transmission in animal models. The D614G substitution conferred increased viral replication in nasal washings and trachea of the upper airway, but not in the lower airway tissues when tested in hamsters (Plante et al., 2020). This correlates with the known comparability of disease severity in humans. The enhanced viral loads in the upper airway increase viral transmission in hamsters and ferrets (Hou et al., 2020a). D, The D614G mutation confers an increased "open" conformation of the spike protein. The D614G mutation, located in the S1 subunit outside of the RBD, disrupts hydrogen bonding between amino acid 614 (indicated by a star) and amino acid T859 of the two adjacent spike protomers, promoting the RDB in an "open" conformation. This in turn increases the ability of the RBD to bind to the ACE2 receptor. The left image (with all three RBD in "closed" conformation) and the right image (with one RBD in "open" conformation) are derived from PDB 6VXX and 6VSB, respectively (Yurkovetskiy et al., 2020). Some graphics were prepared with components from Servier Medical ART (smart.servier.com).

cleavage sites (Jaimes et al., 2020). The S1 subunit is like the "head" and contains the receptor binding domain (RBD) (Yurkovetskiy et al., 2020). The RBD is in either "open" or "closed" conformation. In the "open" conformation, the RBD can bind ACE2 receptor. The S2 subunit is like the "stalk" upon which S1 subunit sits. When the RBD binds ACE2 receptor and S1/S2 cleavage occurs, the S1 subunit dissociates from S2. This causes a conformational change of S2 that projects its fusion peptide to fuse the viral envelope with the cell membrane (Yurkovetskiy et al., 2020). The D614G mutation is located in the S1 subunit outside of the RBD (Figure 1D). The D614G mutation disrupts hydrogen bond formation with amino acid T859 between two adjacent spike protomers, promoting RBD in an "open" conformation (Omotuyi et al., 2020). The increased "open" conformation of the G614 spike increases ACE2 receptor binding. Biolayer interferometry analysis showed that the S1 from the G614 variant had a 2-fold higher affinity to
ACE2 than the S1 from the D614 virus; this higher affinity was maintained in the context of full-length spike protein (Zhou et al., 2020a). Scanning and transmission electron microscopic analysis showed no difference in virion morphology between the G614 and D614 viruses on the infected human airway cultures (Hou et al., 2020b). Taken together, these studies demonstrate that the D614G mutation confers increased ACE2 receptor binding without altering spike cleavage or shedding. This contrasts the earlier data collected in pseudovirus systems, which lack other viral structural proteins of SARS-CoV-2 (e.g., membrane, envelope, and nucleocapsid proteins). The earlier pseudovirus data suggested that the D614G mutation conveyed decreased S1 shedding, increased incorporation of the S protein into the pseudovirion, and increased resistance to spike cleavage (Daniloski et al., 2020; Zhang et al., 2020). This discrepancy highlights the importance of using authentic SARS-CoV-2 for functional studies. 


\section{Potential effect on vaccine and therapeutic anti- body}

Exceptional vaccine and therapeutic antibody development efforts have been ongoing at an unprecedented pace to combat the pandemic (Walsh et al., 2020). Most major vaccines and therapeutic antibodies have been using the original D614 spike sequence (Wang et al., 2020). The D614G mutation calls the vaccine efficacy into concern: is the circulating G614 virus equally susceptible to the antibodies elicited by the D614 spike-based vaccine? Three studies have addressed this question by using highly quantitative reporter SARS-CoV-2 neutralization assays. The first study used a mNeonGreen SARS-CoV-2 neutralization assay (Muruato et al., 2020; Xie et al., 2020) to analyze a panel of sera from hamsters that were immunized with D614 virus. The G614 virus had an average 1.7-fold higher neutralization titer than the D614 virus (Plante et al., 2020). The second study used a luciferase SARS-CoV-2 neutralization assay to show equivalent sensitivities of the D614 and G614 viruses to 25 convalescent human sera; however, the viral genotypes of the 25 patient infections (D614 or G614) were unknown (Hou et al., 2020b). The third study analyzed convalescent sera from COVID-19 patients found that, regardless of whether the infecting virus had a D614 or G614 spike, neutralization titers against G614 pseudoviruses were 1.9- to 2.0-fold higher than those against D614 pseudoviruses (Weissman et al., 2020). This same study also examined sera from animals and humans who were vaccinated with mRNA candidates encoding distinct versions of the D614 spike antigen. The results showed that pseudovirus neutralization titers against G614 pseudovirus were 3.9- to 6.5-fold higher than those against D614 pseudovirus. Although the results vary from different assays and laboratories, the differences are not biologically significant, indicating that the D614G mutation does not compromise the neutralizing activity of the antibodies elicited by the D614 spike-based vaccines. This conclusion is further bolstered by the promising data from the ongoing vaccine clinical trials against current strains, which epidemiologically is more likely to be the G614 variant.

As of January 2021, there are two COVID-19 vaccines granted Emergency Use Authorization by the United States Food and Drug Administration: the Pfizer/BioNTech and the Moderna/National Institute of Allergy and Infectious Diseases vaccines. Both vaccine candidates are mRNA vaccines that encode the full-length SARS-CoV-2 S-2P, which has proline substitutions at residues K986 and V987 of the spike protein (Dai and Gao, 2020). The S-2P mutant has been found to generate higher $\mathrm{nAb}$ titers in mice than wildtype spike protein (Dai and Gao, 2020). The two proline substitutions are thought to stabilize the pre-fusion conformation of the spike protein (Dai and Gao, 2020). Besides using the full-length spike protein, an alternative vaccine approach is to directly target the RBD to elicit nAbs that interfere with the RBD-ACE2 interaction. Clinical results are needed to demonstrate the advantage of the RBD-based vaccine candidates. As the D614G mutation is not a part of the RBD, this mutation is not expected to affect the efficacy of RBD-targeted vaccine candidates.

For monoclonal antibodies (mAb), two separate groups using mAbs against six (Hou et al., 2020b) and 10 (Plante et al., 2020) different epitopes of the RBD found similar potency against D614 and G614 reporter SARS-CoV-2; however, one $\mathrm{mAb}$ tested had a 2.1-fold higher potency against the G614 than the D614 virus (Plante et al., 2020). The latter result supports the idea that the D614G mutation causes a conformational change of the spike protein that may affect neutralization in an epitope-specific manner. This is because D614G is outside the RBD and thus this mutation can only allosterically affect the antiviral activities of mAbs targeting the RBD.

\section{Other emerging variants of SARS-CoV-2}

In December 2020, separate highly transmissible, independently developing variants of SARS-CoV-2 were identified as the cause of accelerating case numbers in the United Kingdom (UK) (Rambaut et al., 2020) as well as South Africa (SA) (Tegally et al., 2020). The UK variant, also known as the B.1.1.7 lineage, has multiple mutations, including several potentially biologically relevant mutations within the spike protein (Rambaut et al., 2020). Similarly, the SA variant, also known as the 501Y.V2 lineage, has multiple biologically relevant mutations within the spike protein and three (K417N, E484K, and N501Y) in the RBD (Tegally et al., 2020). The UK and SA variants both independently developed a N501Y mutation located in the RBD of the spike protein. This mutation, additionally enhanced by the other co-mutations that developed, may account for a greater than $50 \%$ increase in transmissibility, based on epidemiologic observations (Davies et al., 2020). However, experiments are needed to confirm these important, epidemiological findings. Similar to the D614G mutation, these new variants beg the question of whether current vaccines in development and use will continue to confer adequate protection. In a study that used sera from 20 individuals who previously received the Pfizer/BioNTech vaccine, $\mathrm{nAb}$ titers were found to be equivalent between the N501 and Y501 variants (Xie et al., 2021). These promising data seem to support that, despite higher transmissibility, infection by the newly emerging mutants would still be prevented by current EUA vaccines. However, caution should be taken as the N501Y study only included a single mutation (Xie et al., 2021). A comprehensive investigation to include the other mutations, parti- 
cularly in combination, is urgently needed. Emerging variants will need to be identified early and monitored vigilantly using genome sequencing studies. Case numbers may rise rapidly, and utmost care must be taken to ensure that current vaccines in development can confer sufficient protection.

\section{Conclusions and future directions}

Both clinical and experimental results support that the D614G mutation increases viral replication in the upper respiratory airway, leading to enhanced transmissibility of SARS-CoV-2; however, this mutation does not increase disease severity, possibly due to comparable levels of D614 and G614 viruses in lungs. The increased viral replication is likely driven by the mutation-mediated "open" conformation of RBD, resulting in enhanced ACE2 binding and virus entry. Based on the neutralizing antibody results, the D614G mutation is not likely to compromise the vaccine efficacy currently under development, whereas the potency of therapeutic mAbs may be affected by the mutation in an epitopedependent manner. The results underscore the importance of using the most up-to-date viral sequence for vaccine development and the necessity of epidemiological surveillance of emerging viruses for monitoring mutations within the global population. Once herd immunity is established through global vaccination, the virus will evolve under a different selective pressure in human population. Finally, the emergence of D614G mutation was accompanied by three other substitutions: C-to-T at nucleotide position 241 in the $5^{\prime}$ untranslated region, a synonymous $\mathrm{C}$-to-T at position 3037 in the NSP3 gene, and a non-synonymous C-to-G at position 14408 in the RNA polymerase gene (Korber et al., 2020). Future studies are required to investigate the biological function of these co-emerged mutations, as well as mutations in the other emerging lineages.

Compliance and ethics The author(s) declare that they have no conflict of interest.

\section{Acknowledgements We thank colleagues for helpful discussion during} this study.

\section{References}

Chan, J.F.W., Zhang, A.J., Yuan, S., Poon, V.K.M., Chan, C.C.S., Lee, A.C. Y., Chan, W.M., Fan, Z., Tsoi, H.W., Wen, L., et al. (2020). Simulation of the clinical and pathological manifestations of coronavirus disease 2019 (COVID-19) in a Golden Syrian Hamster Model: implications for disease pathogenesis and transmissibility. Clin Infect Dis, doi: 10.1093/ cid/ciaa325.

Dai, L., and Gao, G.F. (2020). Viral targets for vaccines against COVID-19. Nat Rev Immunol, doi: 10.1038/s41577-020-00480-0.

Daniloski, Z., Guo, X., and Sanjana, N.E. (2020). The D614G mutation in SARS-CoV-2 Spike increases transduction of multiple human cell types. bioRxiv, doi: 10.1101/2020.06.14.151357.
Davies, N.G., Barnard, R.C., Jarvis, C.I., Kucharski, A.J., Munday, J., Pearson, C.A.B., Russell, T.W., Tully, D.C., Abbott, S., Gimma, A., et al. (2020). Estimated transmissibility and severity of novel SARS-CoV2 Variant of Concern 202012/01 in England. medRxiv, doi: 10.1101/ 2020.12.24.20248822.

Hou, Y.J., Chiba, S., Halfmann, P., Ehre, C., Kuroda, M., Dinnon Iii, K.H., Leist, S.R., Schäfer, A., Nakajima, N., Takahashi, K., et al. (2020a). SARS-CoV-2 D614G variant exhibits efficient replication ex vivo and transmission in vivo. Science eabe8499.

Hou, Y.J., Okuda, K., Edwards, C.E., Martinez, D.R., Asakura, T., Dinnon Iii, K.H., Kato, T., Lee, R.E., Yount, B.L., Mascenik, T.M., et al. (2020b). SARS-CoV-2 reverse genetics reveals a variable infection gradient in the respiratory tract. Cell 182, 429-446.e14.

Huang, C., Wang, Y., Li, X., Ren, L., Zhao, J., Hu, Y., Zhang, L., Fan, G., $\mathrm{Xu}, \mathrm{J}$., Gu, X., et al. (2020). Clinical features of patients infected with 2019 novel coronavirus in Wuhan, China. Lancet 395, 497-506.

Imai, M., Iwatsuki-Horimoto, K., Hatta, M., Loeber, S., Halfmann, P.J., Nakajima, N., Watanabe, T., Ujie, M., Takahashi, K., Ito, M., et al. (2020). Syrian hamsters as a small animal model for SARS-CoV-2 infection and countermeasure development. Proc Natl Acad Sci USA 117, 16587-16595.

Jaimes, J., Millet, J., and Whittaker, G. (2020). Proteolytic cleavage of the SARS-CoV-2 spike protein and the role of the novel S1/S2 site. SSRN J, doi: 10.2139/ssrn.3581359.

Korber, B., Fischer, W.M., Gnanakaran, S., Yoon, H., Theiler, J., Abfalterer, W., Hengartner, N., Giorgi, E.E., Bhattacharya, T., Foley, B., et al. (2020). Tracking Changes in SARS-CoV-2 Spike: Evidence that D614G Increases Infectivity of the COVID-19 Virus. Cell 182, 812-827.e19.

Lorenzo-Redondo, R., Nam, H.H., Roberts, S.C., Simons, L.M., Jennings, L.J., Qi, C., Achenbach, C.J., Hauser, A.R., Ison, M.G., Hultquist, J.F., et al. (2020). A unique clade of SARS-CoV-2 viruses is associated with lower viral loads in patient upper airways. medRxiv, doi: 10.1101/ 2020.05.19.20107144.

Muruato, A.E., Fontes-Garfias, C.R., Ren, P., Garcia-Blanco, M.A., Menachery, V.D., Xie, X., and Shi, P.Y. (2020). A high-throughput neutralizing antibody assay for COVID-19 diagnosis and vaccine evaluation. Nat Commun 11, 4059.

Omotuyi, I.O., Nash, O., Ajiboye, O.B., Iwegbulam, C.G., Oyinloye, E.B., Oyedeji, O.A., Kashim, Z.A., and Okaiyeto, K. (2020). Atomistic simulation reveals structural mechanisms underlying D614G spike glycoprotein-enhanced fitness in SARS-CoV-2. J Comput Chem 41, 2158-2161.

Plante, J.A., Liu, Y., Liu, J., Xia, H., Johnson, B.A., Lokugamage, K.G., Zhang, X., Muruato, A.E., Zou, J., Fontes-Garfias, C.R., et al. (2020). Spike mutation D614G alters SARS-CoV-2 fitness. Nature, doi: 10.1038/s41586-020-2895-3.

Rambaut, A., Loman, N., Pybus, O., Barclay, W., Barrett, J., Carabelli, A., Connor, T., Peacock, T., Robertson, D.L., Volz, E. (2020). Preliminary genomic characterisation of an emergent SARS-CoV-2 lineage in the UK defined by a novel set of spike mutations. Virological, https:// virological.org/t/preliminary-genomic-characterisation-of-an-emergentsars-cov-2-lineage-in-the-uk-defined-by-a-novel-set-of-spike-mutations $/ 563$.

Shang, J., Wan, Y., Luo, C., Ye, G., Geng, Q., Auerbach, A., and Li, F. (2020). Cell entry mechanisms of SARS-CoV-2. Proc Natl Acad Sci USA $117,11727-11734$.

Smith, E.C., Blanc, H., Surdel, M.C., Vignuzzi, M., and Denison, M.R. (2013). Coronaviruses lacking exoribonuclease activity are susceptible to lethal mutagenesis: evidence for proofreading and potential therapeutics. PLoS Pathog 9, e1003565.

Tegally, H., Wilkinson, E., Giovanetti, M., Iranzadeh, A., Fonseca, V., Giandhari, J., Doolabh, D., Pillay, S., San, E.J., Wibmer, K., et al. (2020). Emergence and rapid spread of a new severe acute respiratory syndrome-related coronavirus 2 (SARS-CoV-2) lineage with multiple spike mutations in South Africa. medRxiv, doi: 10.1101/ 2020.12.21.20248640. 
Walsh, E.E., Frenck Jr., R.W., Falsey, A.R., Kitchin, N., Absalon, J., Gurtman, A., Lockhart, S., Neuzil, K., Mulligan, M.J., Bailey, R., et al. (2020). Safety and immunogenicity of two RNA-based COVID-19 vaccine candidates. N Engl J Med 383, 2439-2450.

Wang, C., Liu, Z., Chen, Z., Huang, X., Xu, M., He, T., and Zhang, Z. (2020). The establishment of reference sequence for SARS-CoV-2 and variation analysis. J Med Virol 92, 667-674.

Weissman, D., Alameh, M.G., de Silva, T., Collini, P., Hornsby, H., Brown, R., LaBranche, C.C., Edwards, R.J., Sutherland, L., Santra, S., et al. (2020). D614G spike mutation increases SARS CoV-2 susceptibility to neutralization. Cell Host Microbe 29, 23-31.e4.

Xie, X., Muruato, A., Lokugamage, K.G., Narayanan, K., Zhang, X., Zou, J., Liu, J., Schindewolf, C., Bopp, N.E., Aguilar, P.V., et al. (2020). An infectious cDNA clone of SARS-CoV-2. Cell Host Microbe 27, 841848.e3.

Xie, X., Zou, J., Fontes-Garfias, C.R., Xia, H., Swanson, K.A., Cutler, M., Cooper, D., Menachery, V.D., Weaver, S., Dormitzer, P.R., et al. (2021). Neutralization of N501Y mutant SARS-CoV-2 by BNT162b2 vaccineelicited sera. Res Sq, rs.3.rs-143532.
Yurkovetskiy, L., Wang, X., Pascal, K.E., Tomkins-Tinch, C., Nyalile, T.P., Wang, Y., Baum, A., Diehl, W.E., Dauphin, A., Carbone, C., et al. (2020). Structural and functional analysis of the D614G SARS-CoV-2 spike protein variant. Cell 183, 739-751.e8.

Zhang, L., Jackson, C.B., Mou, H., Ojha, A., Rangarajan, E.S., Izard, T., Farzan, M., Choe, H. (2020). The D614G mutation in the SARS-CoV-2 spike protein reduces $\mathrm{S} 1$ shedding and increases infectivity. bioRxiv, doi: $10.1101 / 2020.06 .12 .148726$.

Zhou, B., Thao, T.T.N., Hoffmann, D., Taddeo, A., Ebert, N., Labroussaa, F., Pohlmann, A., King, J., Portmann, J., Halwe, N.J., et al. (2020a). SARS-CoV-2 spike D614G variant confers enhanced replication and transmissibility. bioRxiv, doi: 10.1101/2020.10.27.357558.

Zhou, P., Yang, X.L., Wang, X.G., Hu, B., Zhang, L., Zhang, W., Si, H.R., Zhu, Y., Li, B., Huang, C.L., et al. (2020b). A pneumonia outbreak associated with a new coronavirus of probable bat origin. Nature 579, 270-273.

Zhu, N., Zhang, D., Wang, W., Li, X., Yang, B., Song, J., Zhao, X., Huang, B., Shi, W., Lu, R., et al. (2020). A novel coronavirus from patients with pneumonia in China, 2019. N Engl J Med 382, 727-733. 